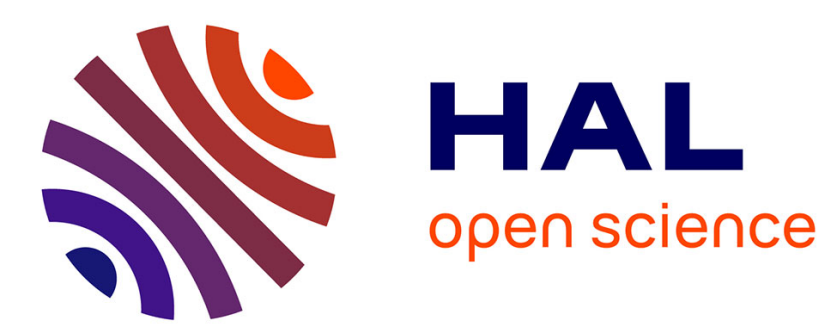

\title{
On the continuous contact force models for soft materials in multibody dynamics
}

Paulo Flores, Margarida Machado, Miguel Silva, Jorge Martins

\section{To cite this version:}

Paulo Flores, Margarida Machado, Miguel Silva, Jorge Martins. On the continuous contact force models for soft materials in multibody dynamics. Multibody System Dynamics, 2011, 25, pp. 357375. 10.1007/s11044-010-9237-4 . hal-01333699

\section{HAL Id: hal-01333699 https://hal.science/hal-01333699}

Submitted on 18 Jun 2016

HAL is a multi-disciplinary open access archive for the deposit and dissemination of scientific research documents, whether they are published or not. The documents may come from teaching and research institutions in France or abroad, or from public or private research centers.
L'archive ouverte pluridisciplinaire HAL, est destinée au dépôt et à la diffusion de documents scientifiques de niveau recherche, publiés ou non, émanant des établissements d'enseignement et de recherche français ou étrangers, des laboratoires publics ou privés. 


\title{
On the continuous contact force models for soft materials in multibody dynamics
}

\author{
Paulo Flores • Margarida Machado • Miguel T. Silva • Jorge M. Martins
}

\begin{abstract}
A general and comprehensive analysis on the continuous contact force models for soft materials in multibody dynamics is presented throughout this work. The force models are developed based on the foundation of the Hertz law together with a hysteresis damping parameter that accounts for the energy dissipation during the contact process. In a simple way, these contact force models are based on the analysis and development of three main issues: (i) the dissipated energy associated with the coefficient of restitution that includes the balance of kinetic energy and the conservation of the linear momentum between the initial and final instant of contact; (ii) the stored elastic energy, representing part of initial kinetic energy, which is evaluated as the work done by the contact force developed during the contact process; (iii) the dissipated energy due to internal damping, which is evaluated by modeling the contact process as a single degree-of- freedom system to obtain a hysteresis damping factor. This factor takes into account the geometrical and material properties, as well as the kinematic characteristics of the contacting bodies. This approach has the great merit that can be used for contact problems involving materials with low or moderate values of coefficient of restitution and, therefore, accommodate high amount of energy dissipation. In addition, the resulting contact force model is suitable to be included into the equations of motion of a multibody system and contributes to their stable numerical resolution. A demon-
\end{abstract}


strative example of application is used to provide the results that support the analysis and discussion of procedures and methodologies described in this work.

Keywords Contact force - Continuous analysis - Soft materials · Coefficient of restitution Elastic energy $\cdot$ Internal damping $\cdot$ Multibody dynamics

\section{Introduction}

Over the last century, the academic and scientific communities have demonstrated an increasing interest in solving problems related to contact-impact phenomena in mechanical multibody systems. Undeniably, the classical problem of the contact mechanics is a quite old topic in engineering applications [1,2]. The pioneering work on the frictionless collision between rigid bodies was due to Hertz who developed the theory with his name during the Christmas vacation of 1880 at the age of twenty-three [3]. The contact problem has been an intensive topic of research over the last decades and deserved the attention of many authors, which led to the development of relevant work and even to the publication of a good number of textbooks totally devoted to this issue, such as those by Goldsmith [4], Brach [5], Pfeiffer and Glocker [6], Johnson [7], Stronge [8], Wriggers [9], among others. The contactimpact study in multibody dynamics has received a great deal of attention in the past few years and it still remains an active field of research and development [10-22].

Contact-impact events can frequently occur in multibody systems, and in many cases the function of mechanical systems is based on them. Gilardi and Sharf [23] describe an impact as a complex physical phenomenon for which the main characteristics are a very short duration, high force levels, rapid energy dissipation, and large changes in the velocities of bodies. In turn, contact implies a continuous process which takes place over a finite time. The analysis of contact between two bodies can be extended to the analysis of collision in a multibody system. Whenever a body in a multibody system experiences a hard stop, contact forces of complex nature act on the body and the corresponding impulse is transmitted throughout the system.

The bodies that belong to a multibody system can be considered as rigid or flexible. A body is said to be rigid when its deformations are assumed to be small such that they do not influence the global motion produced by the body. The expression flexible multibody system refers to a system holding deformable bodies with internal dynamics [24]. In fact, rigid bodies are a representation of reality because bodies are not absolutely rigid in nature. However, many physical mechanical systems contain a combination of rigid and flexible bodies, or rigid bodies with soft surfaces, or bodies that are rigid enough to be considered rigid overall, but soft enough to experience significant local deformations during contact. In this case, the contact approach must be able to model the dynamics of contact between compliant surfaces.

The compliant continuous contact force models, commonly referred as penalty methods, have been gaining significant importance in the context of multibody systems with contacts due to their computational simplicity and efficiency. In these models, the contact force is expressed as a continuous function of the relative deformation of the contacting surfaces during the complete period of contact. In addition, most of the continuous contact force models contribute to an efficient integration of the equations of motion and account for some level of energy dissipation. This amount of good reasons explains why some of the most popular commercial codes use the penalty methods to treat contact problems [25-27].

The energy loss due to the contact process in the normal direction can be expressed in terms of the coefficient of restitution. This parameter has different definitions, being one of 
the most popular and commonly used the Newton's law of restitution, also known as kinematical coefficient of restitution. In a simple manner, this definition states that when two bodies collide, the post-impact velocity is related to the preimpact velocity by a constant of proportionality called coefficient of restitution [28]. This coefficient is, in general, assumed to be constant; however, it is dependent on several factors such as the geometry of the contacting surfaces, preimpact velocity, local material properties, duration of the contact, temperature, and friction $[4,5,29]$. The analysis and discussion of these issues is out of the scope of the present work, being the interested reader in this particular topic referred to the work by Gilardi and Sharf [23].

The initial kinetic energy produced in a contact event is converted in different measures during the contact process. First of all, part of the initial kinetic energy is transformed into elastic strain energy which, in turn, is converted in the final kinetic energy at the end of the contact. The remaining amount of kinetic energy is dissipated during the contact process in several forms such as wave propagation [8, 30], material damping [4, 31], plasticity $[5,32]$, and heat $[23,33]$. Stronge [8] demonstrated that stress and deformation waves do not contribute to energy dissipation when the size and material properties of the contacting bodies are similar. Shivaswamy [34] showed that at low impact velocities, the damping force component is the prime factor for energy dissipation. At high impact velocities, that is, those exceeding the propagation velocity of elastic deformation waves are likely to dissipate energy in a form of permanent local deformation.

The general motivation for this work comes from current interest in developing mathematical models for the dynamics of constrained multibody systems involving contact events between soft materials, such as in the case of biomechanical systems and bushing elements made with polymer materials [35-41]. In fact, modeling contacts with a high degree of energy dissipation plays a crucial role in the analysis, design and control of many mechanical systems in the measure that soft materials are characterized by low or medium values of the coefficient of restitution. The methodology adopted in this work follows closely that of $[42,43]$, in which an explicit relation between the coefficient of restitution and a hysteresis damping factor is derived. Furthermore, the approach presented in this study is based on the analysis of dissipated energy related to the coefficient of restitution, stored elastic energy, and the dissipated energy associated with the internal damping of the contacting bodies. The methodology and procedures adopted throughout this work are presented and analyzed with the help of a numerical simulation of the classical bouncing ball problem.

\section{General issues of continuous contact force models}

The theory developed by Hertz remains the foundation for most contact problems encountered in engineering applications. The Hertz law relates the contact force with a nonlinear power function of deformation and is expressed as [44]

$$
F_{N}=K \delta^{n}
$$

where $K$ represents the generalized stiffness parameter and $\delta$ is the relative normal deformation between the contacting bodies. The exponent $n$ is typically equal to $3 / 2$ [2]. The generalized parameter $K$ is dependent on the material properties and the shape of the surfaces in contact. For two spheres $i$ and $j$ in contact, the generalized parameter is a function of radii of the spheres and the material properties as [4]

$$
K=\frac{4}{3\left(\sigma_{i}+\sigma_{j}\right)}\left[\frac{R_{i} R_{j}}{R_{i}+R_{j}}\right]^{\frac{1}{2}}
$$


in which the material parameters $\sigma_{i}$ and $\sigma_{j}$ are given by

$$
\sigma_{l}=\frac{1-v_{l}^{2}}{E_{l}}, \quad(l=i, j)
$$

and the quantities $v_{l}$ and $E_{l}$ are the Poisson's ratio and Young's modulus associated with each sphere, respectively.

It is apparent that the Hertz contact law given by (1) is limited to contact with elastic deformations and does not include the damping effect. This force model represents the contact process as a nonlinear spring along the direction of collision. Although the Hertz law is based on the elasticity theory [45], some studies have been performed to extend its application to include energy dissipation. An alternative contact force approach that accounts for the energy loss during the contact process is the Kelvin-Voigt model. This force model considers a linear spring in conjunction with a linear damper and can be expressed as [46]

$$
F_{N}=K \delta+D \dot{\delta}
$$

where the first term of the right-hand side is referred to as the elastic force and the second term accounts for the energy dissipation during the contact process. In (4), $D$ is a hysteresis coefficient and $\dot{\delta}$ represents the relative normal contact velocity, having the remaining variables the same meaning as it was described above.

The constitutive law given by (4) presents some weaknesses, namely the fact that the contact force at zero deformation is not continuous due to the existence of the damping component. This particular issue is not realistic because when the contact begins, both elastic and damping force components must be null. Moreover, at the end of the contact, the deformation is null, the relative contact velocity is negative and, consequently, the resulting contact force is also negative, which does not make sense from the physical point of view because the bodies can not attract each other [3, 47, 48].

Hunt and Crossley [31] showed that the linear spring-damper model does not represent the physical nature of energy transferred during the contact. Instead, they represented the contact force by the Hertz force-deformation law with a nonlinear viscous-elastic element as

$$
F_{N}=K \delta^{n}+\chi \delta^{n} \dot{\delta}
$$

where $\chi$ is called the hysteresis damping factor given by

$$
\chi=\frac{3 K\left(1-c_{r}\right)}{2 \dot{\delta}^{(-)}}
$$

in which $K$ represents the generalized stiffness parameter, $c_{r}$ is the coefficient of restitution and $\dot{\delta}^{(-)}$is the initial contact velocity. After some mathematical manipulation, the expression for this contact force model has the following form [18]:

$$
F_{N}=K \delta^{n}\left[1+\frac{3\left(1-c_{r}\right)}{2} \frac{\dot{\delta}}{\dot{\delta}^{(-)}}\right]
$$

With this model, the energy loss during the contact is assumed to be associated with the material damping of the contacting bodies, which would dissipate energy in the form of heat. The Hunt and Crossley force model expresses the damping as a function of deformation, which sounds physically reasonable. Furthermore, this model does not present 
discontinuities at the initial instant of contact and at the end of contact, that is, it starts and ends with the zero value.

Lankarani and Nikravesh [33] used the general trend of the Hertz contact law incorporated with a hysteresis damping factor to propose a continuous force model for the contact analysis of multibody systems. The hysteresis damping factor that accommodates the energy dissipation during the contact is a function of the deformation and is written as

$$
\chi=\frac{3 K\left(1-c_{r}^{2}\right)}{4 \dot{\delta}^{(-)}}
$$

Based on the analysis of Hunt and Crossley [31] that separates the contact force into elastic and dissipative components, the force model proposed by Lankarani and Nikravesh can be expressed as

$$
F_{N}=K \delta^{n}\left[1+\frac{3\left(1-c_{r}^{2}\right)}{4} \frac{\dot{\delta}}{\dot{\delta}^{(-)}}\right]
$$

which is quite similar to the model proposed by Hunt and Crossley. It should be highlighted that in the Lankarani and Nikravesh force model, the parameters have been evaluated from the physics of the problem by equating the kinetic energy loss in the system to the work done by the contact force. Since the kinetic energy loss is in terms of the relative velocity squared, then the expression for the hysteresis damping factor and the contact force include a term in $c_{r}^{2}$.

The contact force models given by (7) and (9) are valid for the cases in which the dissipated energy during the contact is small when compared to the maximum absorbed elastic energy, that is, the relation is valid for the values of the coefficient of restitution close to unity. The consequences of this simplifying premise are discussed in detail by Lankarani and Nikravesh [33]. In a later paper, Lankarani and Nikravesh [32] proposed a new approach for the contact force model, in which the permanent or plastic deformation was also included.

By analyzing (6) and (8), it can be observed that for a perfectly elastic contact, i.e., $c_{r}=1$, the hysteresis damping factor assumes a zero value, while for a perfectly plastic contact, i.e., $c_{r}=0$, the hysteresis damping factor does not assume an infinite value as it was expected. This fact is not surprising because, for instance, Lankarani and Nikravesh [33] derived their model for high values of restitution coefficient, being, therefore, valid for hard contacts, such as those between metals [49-52]. The values of the coefficient of restitution for soft materials are low or medium [40, 43, 48, 53], which clearly indicate that the hysteresis damping factor given by (6) and (8) cannot be used. Therefore, a more accurate expression for this factor is required.

\section{Description of the elastic contact between two solid spheres}

Prior to present the detailed description of the presented approach for the normal contact force, let us first introduce some fundamental issues related to contact mechanics, which play a crucial role since they constitute the basis that supports the general methodology used to develop the main procedures followed in the present work.

Among the different types of contact that can be defined for the collision between two bodies that belong to a multibody system, the one-dimensional direct central contact between two solid spheres is considered here with the purpose to keep the analysis simple. 
When the spheres contact each other, deformation takes place in the local contact area resulting in a contact force. Two different phases can be distinguished during the contact process, namely the compression, approaching or loading period, and the restitution, separating or unloading period $[4,5]$. The first phase starts when the two spheres come in contact and ends when the maximum deformation is reached. During this phase, the relative velocity of the contact points on the two bodies in the normal contact direction is gradually reduced to zero. The end of the compression phase is referred to as the instant of maximum compression or maximum approach, which corresponds to the maximum normal contact force if no dissipation of energy is considered. In turn, the restitution phase starts at this instant of time and ends when the two spheres separate from each other.

Figure 1 illustrates the two different phases of a direct central contact between two solid spheres. The spheres are labeled as bodies $i$ and $j$ and have masses $m_{i}$ and $m_{j}$. The pre- and post-impact velocities associated with each sphere are denoted as $v_{i}^{(-)}, v_{j}^{(-)}, v_{i}^{(+)}$, and $v_{j}^{(+)}$, respectively. At the end of the compression phase, the two spheres move with a common instantaneous velocity represented by $v_{i j}^{(\max )}$.

Figure 2 shows a general diagram for the deformation evolution with time during a single contact between two spheres, as well as the contact force plotted against deformation. In

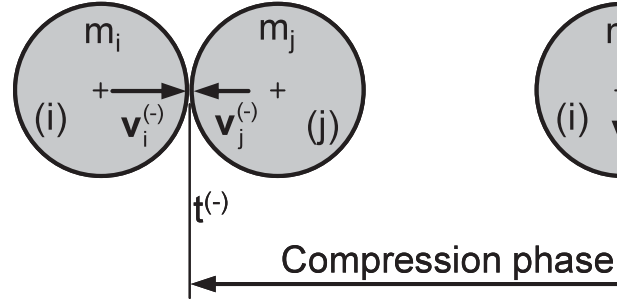

(a)

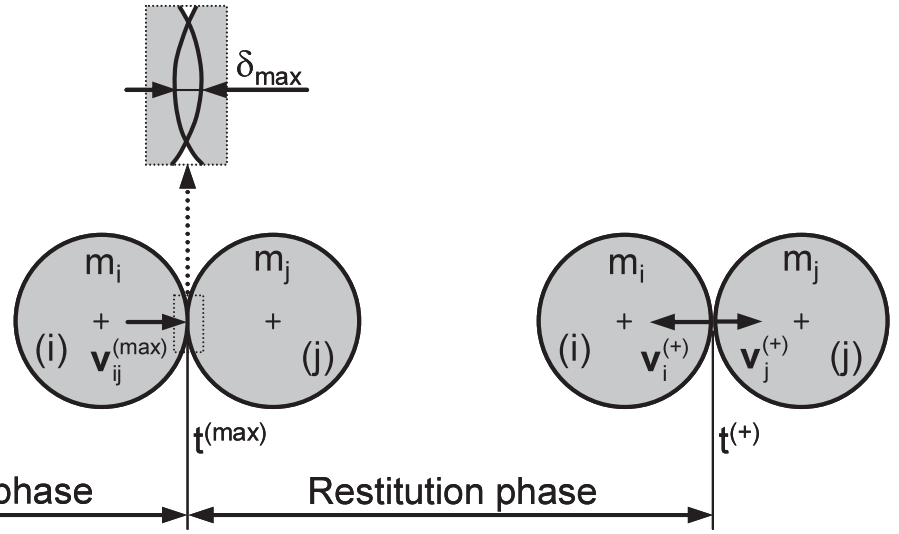

(b) (c)

Fig. 1 Representation of the one-dimensional direct central collision between two solid spheres: (a) Beginning of the compression phase; (b) Maximum approach instant; (c) Termination of the restitution phase

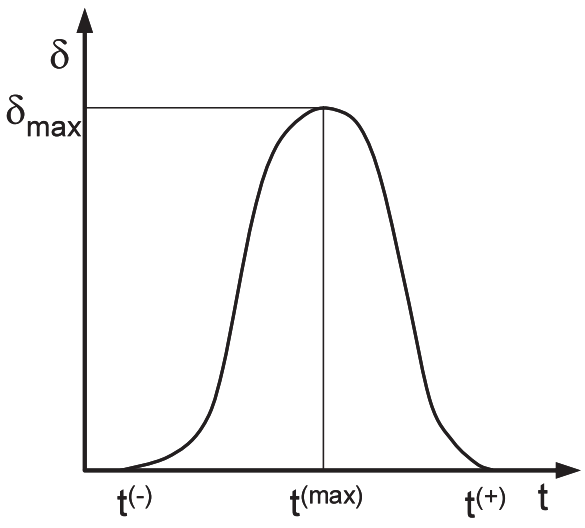

(a)

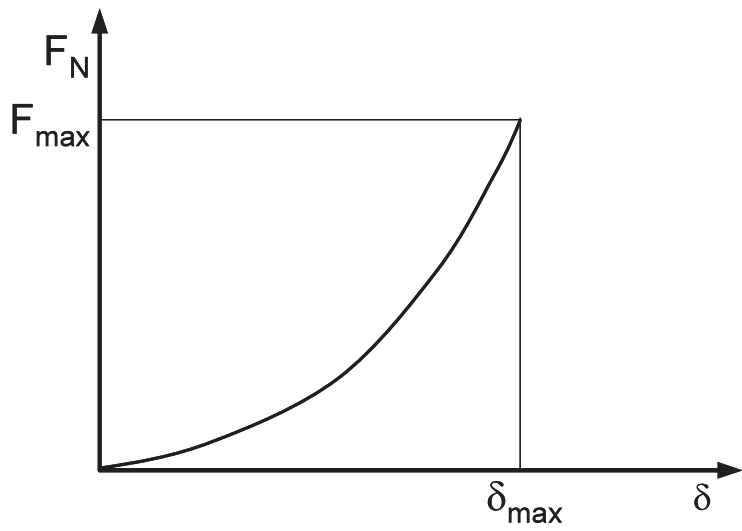

(b)

Fig. 2 Dynamic response of a contact event: (a) Deformation versus time; (b) Contact force versus deformation 
these diagrams, the instant of initial contact, the instant of maximum deformation and the instant of separation are denoted as $t^{(-)}, t^{(\max )}$, and $t^{(+)}$, respectively. In turn, the symbols $\delta_{\max }$ and $F_{\max }$ refer to the values of deformation and contact force at the instant of time $t^{(\max )}$, respectively.

\section{Dissipated energy associated with the coefficient of restitution}

The simplest manner to quantify the energy loss during a contact event is to use the concept of coefficient of restitution, which can be evaluated from the balance of energy that occurs between the initial instant of contact $t^{(-)}$and the final instant of contact $t^{(+)}$. For this purpose, let consider $T^{(-)}$and $T^{(+)}$, respectively, the kinetic energy of the two contacting spheres at the instants of time $t^{(-)}$and $t^{(+)}$. Thus, the energy balance can be expressed as [54]

$$
\Delta E=T^{(-)}-T^{(+)}=\frac{1}{2} m_{i}\left[\left(v_{i}^{(-)}\right)^{2}-\left(v_{i}^{(+)}\right)^{2}\right]+\frac{1}{2} m_{j}\left[\left(v_{j}^{(-)}\right)^{2}-\left(v_{j}^{(+)}\right)^{2}\right]
$$

in which, the post-impact velocities are the unknown quantities.

The linear momentum associated with the two spheres $i$ and $j$ in the direction of the contact is conserved because the contact forces are internal to the system [55]. Therefore, it is possible to write the following equation:

$$
m_{i}\left(v_{i}^{(-)}-v_{i}^{(+)}\right)+m_{j}\left(v_{j}^{(-)}-v_{j}^{(+)}\right)=0
$$

On the other hand, taking advantage of the kinematical or Newton's definition of the coefficient of restitution, which expresses the ratio between the post- and the pre-impact velocities of the spheres in the contact, yields [8]

$$
c_{r}=-\frac{v_{i}^{(+)}-v_{j}^{(+)}}{v_{i}^{(-)}-v_{j}^{(-)}}=-\frac{\dot{\delta}^{(+)}}{\dot{\delta}^{(-)}}
$$

where $\dot{\delta}^{(-)}$and $\dot{\delta}^{(+)}$are, respectively, the relative approaching velocity and the relative separating velocity.

Equations (10)-(12) form a system of three equations with three unknowns $v_{i}^{(+)}, v_{j}^{(+)}$, and $\Delta E$. Solving this system of equations results in

$$
\begin{aligned}
v_{i}^{(+)} & =\frac{1}{m_{i}+m_{j}}\left[\left(m_{i}-c_{r} m_{j}\right) v_{i}^{(-)}+\left(1+c_{r}\right) m_{j} v_{j}^{(-)}\right] \\
v_{j}^{(+)} & =\frac{1}{m_{i}+m_{j}}\left[\left(m_{j}-c_{r} m_{i}\right) v_{j}^{(-)}+\left(1+c_{r}\right) m_{i} v_{i}^{(-)}\right] \\
\Delta E & =\frac{1}{2} \frac{m_{i} m_{j}}{m_{i}+m_{j}}\left(v_{i}^{(-)}-v_{j}^{(-)}\right)^{2}\left(1-c_{r}^{2}\right)
\end{aligned}
$$

Equation (15) represents the dissipated energy in the contact process of two solid spheres in terms of the coefficient of restitution and relative approaching velocity, which can be simplified in the following form:

$$
\Delta E=\frac{1}{2} m\left(\dot{\delta}^{(-)}\right)^{2}\left(1-c_{r}^{2}\right)
$$


where $m$ is the equivalent mass of the two spheres given by

$$
m=\frac{m_{i} m_{j}}{m_{i}+m_{j}}
$$

The coefficient of restitution describes the type of collision. For a fully elastic contact the restitution coefficient is equal to unity, while for a fully plastic contact the restitution coefficient is null. The most general and predominant type of collision is the oblique eccentric collision, which involves both relative normal velocity and relative tangential velocity $[56,57]$.

\section{Stored elastic strain energy during contact}

As it was mentioned above, at the end of the compression phase, the two spheres move with a common instantaneous velocity $v_{i j}^{(\max )}$. Moreover, the initial kinetic energy associated with the contact of the two spheres has three different destinations. One part is transformed into the kinetic energy of the two spheres moving with the same common velocity. A second part is converted to stored elastic strain energy. Finally, a third part of the initial kinetic energy is dissipated. Thus, the balance of energy between the start and end of the compression phase can be written as follows:

$$
T^{(-)}=T^{(\max )}+U^{(\max )}+\Delta E_{c}
$$

in which $T^{(\max )}$ represents the kinetic energy of the system at the end of the compression phase, $U^{(\max )}$ is the maximum elastic strain energy stored and $\Delta E_{c}$ denotes the dissipated energy associated with internal damping of the material. This last term will be discussed in detail in the next section.

The stored strain energy is equal to the work done by the contact force that develops from the state of zero deformation to the state of maximum deformation, that is [33]

$$
U^{(\max )}={ }_{0}^{\delta_{\max }} F_{N} \mathrm{~d} \delta
$$

In order to obtain a solution of this integral let consider that the contact force is represented by the pure elastic Hertz law (1), and consequently, the elastic strain energy absorbed can be rewritten as

$$
U^{(\max )}=\int_{0}^{\delta_{\max }} K \delta^{\frac{3}{2}} \mathrm{~d} \delta
$$

Finally, the stored elastic strain energy is given by

$$
U^{(\max )}=\frac{2}{5} K \delta_{\max }^{\frac{5}{2}}
$$

\section{Dissipated energy due to internal damping}

In this section, the dissipated energy due to internal damping is determined. With the purpose of reaching this desideratum, let consider an equivalent system to the contact process 
Fig. 3 (a) Contact between two rigid spheres; (b) Equivalent system

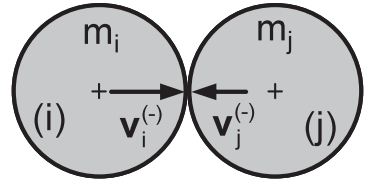

(a)

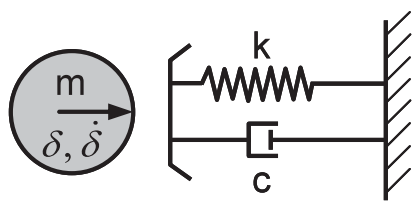

(b)

between two spheres materialized by a single degree of freedom dynamic system, as depicted in Fig. 3. In this model, the initial deformation between the spheres is zero and the relative initial contact velocity is denoted by $\dot{\delta}^{(-)}$. The mathematical representation of this dynamic system can be expressed as $[5,58,59]$

$$
m \ddot{\delta}+c \dot{\delta}+k \delta=0
$$

where $m$ is the equivalent mass given by (17), $c$ represents the viscous damping coefficient and $k$ is the equivalent stiffness. The main reason to use the model of (22) owes to the fact that it matches precisely with the ideal Kelvin-Voigt viscoelastic approach [4].

Equation (22) is a second-order differential equation with constant coefficients; the solution of which is given by [58]

$$
\begin{aligned}
& \delta=e^{-\zeta \omega t} \frac{\dot{\delta}^{(-)}}{\omega_{d}} \sin \left(\omega_{d} t\right) \\
& \dot{\delta}=e^{-\zeta \omega t} \dot{\delta}^{(-)} \cos \left(\omega_{d} t\right)-\zeta e^{-\zeta \omega t} \frac{\omega \dot{\delta}^{(-)}}{\omega_{d}} \sin \left(\omega_{d} t\right)
\end{aligned}
$$

where $\zeta$ denotes the damping ratio, $\omega$ is the radial frequency and $\omega_{d}$ represents the damped frequency. These three parameters are expressed as

$$
\begin{aligned}
\zeta & =\frac{c}{2 m \omega} \\
\omega & =\frac{\bar{k}}{m} \\
\omega_{d} & =\omega \overline{1-\zeta^{2}}
\end{aligned}
$$

In the case that the damping effect is neglected, the values of $t^{(\max )}$ and $\delta_{\max }$ are given by

$$
\begin{aligned}
\delta_{\max } & =\frac{\dot{\delta}^{(-)}}{\omega} \\
t^{(\max )} & =\frac{\pi}{2 \omega}
\end{aligned}
$$

Substituting (28) and (29) into (23) and (24), and taking advantage of the fundamental law of the trigonometry, yields the following mathematical relation:

$$
\left({\frac{\delta}{\delta_{\max }}}^{2}+{\frac{\dot{\delta}}{\dot{\delta}^{(-)}}}^{2}=1\right.
$$

which is an equation of an ellipse for the deformation and velocity. Thus, it is possible to express the velocity of deformation as function of the deformation for both compression and 
restitution phases, respectively, as

$$
\begin{aligned}
& \dot{\delta}=\dot{\delta}^{(-)} \overline{1-\left(\frac{\delta}{\delta_{\max }}\right.} \\
& \dot{\delta}=\dot{\delta}^{(+)} \overline{1-\left({\frac{\delta}{\delta_{\max }}}^{2}\right.}
\end{aligned}
$$

At this stage, it is important to note that the post-impact velocity $\dot{\delta}^{(+)}$is negative because the spheres are separated from each other.

The dissipated energy is evaluated through the work done by the damping force component, which can be written as [33]

$$
\Delta E=\oint \chi \delta^{\frac{3}{2}} \dot{\delta} \mathrm{d} \delta
$$

where $\oint$ refers to the integral around a hysteresis loop for the contact force [33]. The total dissipated energy due to the damping force associated with the compression and restitution phases can be expressed, respectively, as

$$
\begin{aligned}
& \Delta E_{c}={ }_{0}^{\delta_{\max }} \chi \delta^{\frac{3}{2} \dot{\delta}^{(-)}} \overline{1-\left(\frac{\delta}{\delta_{\max }^{2}}\right.} \mathrm{d} \delta \\
& \Delta E_{r}=\underbrace{\delta_{\max }}_{0} \chi \delta^{\frac{3}{2}}\left|\dot{\delta}^{(+)}\right| \overline{1-\left(\frac{\delta}{\delta_{\max }}\right.}{ }^{2} \mathrm{~d} \delta
\end{aligned}
$$

and consequently, the total amount of dissipated energy is given by

$$
\Delta E=\Delta E_{c}+\Delta E_{r}=\chi\left(\dot{\delta}^{(-)}+\left|\dot{\delta}^{(+)}\right|\right) \int_{0}^{\delta_{\max }} \delta^{\frac{3}{2}} \overline{1-\left(\frac{\delta}{\delta_{\max }}\right.} \mathrm{d} \delta
$$

Applying the integral transformation to (36) yields [43]

$$
\Delta E=\chi\left(\dot{\delta}^{(-)}+\left|\dot{\delta}^{(+)}\right|\right) \delta_{\max }^{\frac{5}{2}} x_{0}^{\frac{3}{2}} \overline{1-x^{2}} \mathrm{~d} x
$$

It should be highlighted that the value of the integral term in (37) can only be evaluated numerically, yielding

$$
\int_{0}^{x^{\frac{3}{2}}} \overline{1-x^{2}} \mathrm{~d} x \simeq \frac{1}{4}
$$

The detailed demonstration of this solution is offered in the appendix presented at the end of this paper. Hence, substituting this value into (37) results in

$$
\Delta E=\frac{1}{4} \chi\left(\dot{\delta}^{(-)}+\left|\dot{\delta}^{(+)}\right|\right) \delta_{\max }^{\frac{5}{2}}
$$

Regarding now (39) and taking into account the definition of the coefficient of restitution (12), then (39) can be rewritten in the following form:

$$
\Delta E=\frac{1}{4} \chi\left(1-c_{r}\right) \dot{\delta}^{(-)} \delta_{\max }^{\frac{5}{2}}
$$


When the dissipated energy during the contact of two spheres is taken into account, the balance of energy given by (18) can be expanded as

$$
\frac{1}{2} m_{i}\left(v_{i}^{(-)}\right)^{2}+\frac{1}{2} m_{j}\left(v_{j}^{(-)}\right)^{2}=\frac{1}{2}\left(m_{i}+m_{j}\right) v_{i j}^{(\max )}+\frac{2}{5} K \delta_{\max }^{\frac{5}{2}}+\frac{1}{4} \chi \dot{\delta}^{(-)} \delta_{\max }^{\frac{5}{2}}
$$

The linear momentum balance between instants $t^{(-)}$and $t^{(\max )}$ is given by

$$
m_{i} v_{i}^{(-)}+m_{j} v_{j}^{(-)}=\left(m_{i}+m_{j}\right) v_{i j}^{(\max )}
$$

Thus, combining (16), (40), (41), and (42), after some basic mathematical manipulation, the hysteresis damping factor $\chi$ can finally be expressed as

$$
\chi=\frac{8 K\left(1-c_{r}\right)}{5 c_{r} \dot{\delta}^{(-)}}
$$

which represents an explicit relation of the equivalent damping factor, the generalized stiffness, the initial contact velocity, and the coefficient of restitution.

From the analysis of (43), it can be concluded that for a perfectly elastic contact, i.e. $c_{r}=1$, the hysteresis damping factor assumes a zero value, and when the contact is purely plastic, i.e., $c_{r}=0$, the hysteresis damping factor is infinite, which is reasonable from physical point of view. Finally, introducing the expression of the hysteresis damping factor given by (43) into (5) yields the expression for normal contact force described in this work

$$
F_{N}=K \delta^{n}\left[1+\frac{8\left(1-c_{r}\right)}{5 c_{r}} \frac{\dot{\delta}}{\dot{\delta}^{(-)}}\right]
$$

\section{Demonstrative application to a bouncing ball}

The example of application considered here is the classical bouncing ball problem, which is one of the simplest contact systems [43, 60]. Figure 4 shows an elastic ball made of PTFE, with an initial height equal to $1.0 \mathrm{~m}$, a mass of $1.0 \mathrm{~kg}$, a moment of inertia equal to $0.1 \mathrm{~kg} \mathrm{~m}^{2}$, a radius $R=0.1 \mathrm{~m}$, and an equivalent stiffness equal to $140 \times 10^{6} \mathrm{~N} / \mathrm{m}^{3 / 2}$. The ball is released from the initial position under the action of gravity only, which is taken to act in the negative $y$ direction. Thus, the ball falls down until it collides with the ground, which

Fig. 4 Bouncing ball example

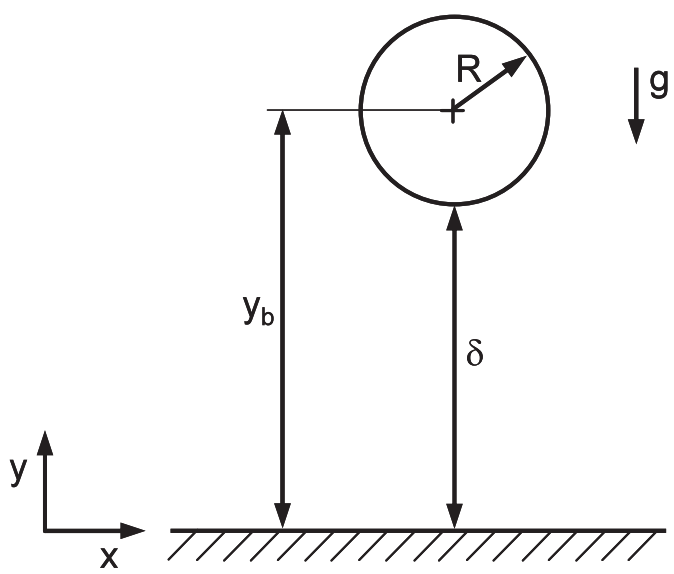




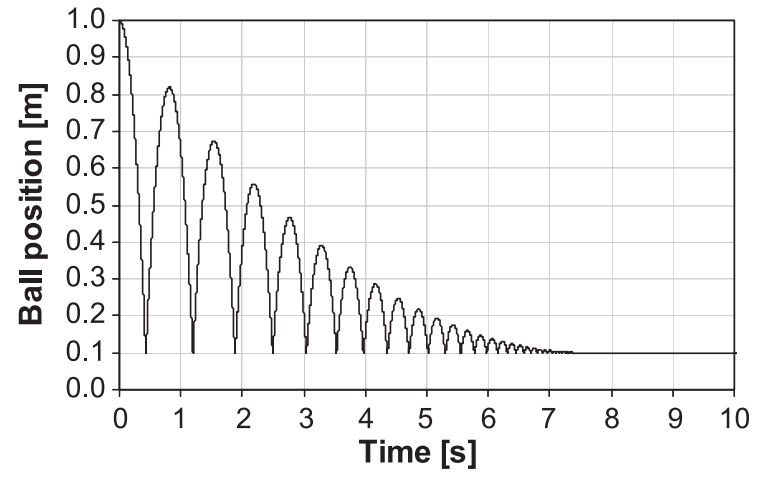

(a)

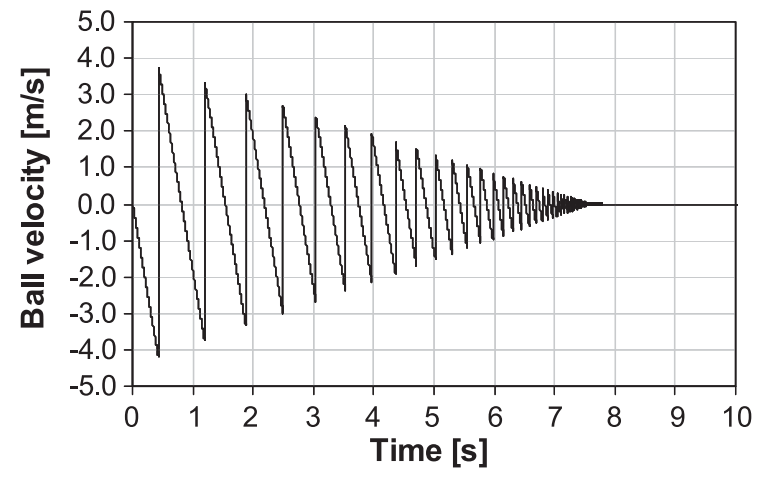

(b)

Fig. 5 Kinematic simulation results of a ball falling on the ground: (a) Ball position; (b) Ball velocity

is considered to be rigid and stationary. When the ball collides with the ground, a contact takes place and the ball rebounds, producing jumps, in which height depends on the value of the coefficient of restitution.

When the ball contact with the ground, the deformation is evaluated as

$$
\delta=y_{b}-R
$$

where $y_{b}$ is the $y$ coordinate of the ball center of mass and $R$ represents the ball radius. Positive values of $\delta$ mean that there is no contact between the ball and ground. Therefore, the detection of the instant of contact occurs when the sign of deformation changes between two discrete instants in time [61]. This problem was analyzed by using a FORTRAN code named MUBODYNA (acronym for Multibody Dynamics) [62].

Figure 5 includes the global results of the ball kinematics, namely the ball position and velocity, when the coefficient of restitution is equal to 0.9 and the contact force model described in this paper is considered. When the ball hits the ground, a contact occurs and the ball is rebounded. The restitution coefficient is less than unit, therefore, the ball jumps less high after each contact. The time lapse between the contacts tends to zero and ends up when the ball remains on the ground.

Figures $6 \mathrm{a}-\mathrm{c}$ show the time plots of the deformation, velocity of deformation and contact force developed during the first contact between the ball and the ground. Additionally, Fig. $6 \mathrm{~d}$ illustrates the contact force plotted against the deformation. In the case studies presented here, five different values for the coefficient of restitution are selected, namely 1.0, $0.8,0.6,0.4$, and 0.2. As it was expected, when the coefficient of restitution decreases, the maximum deformation and the maximum contact force values are reduced. These facts are accompanied with the increase of the contact duration and the hysteresis loop, which in turn is associated with the energy dissipated during the contact process. When the coefficient of restitution is equal to unit, which corresponds to the pure Hertz contact law, there is no energy dissipation in the contact process. This fact is evident in the force-deformation diagram of Fig. 6d, which does not present a hysteresis loop. In general, the global data produced here are corroborated by similar analysis available in the thematic literature $[11,33,43]$.

A similar analysis presented by Lankarani and Nikravesh [33] is also included in the present study, in which the post-impact velocity that results from the first impact is used to compute the amount of restitution produced by the contact force. Thus, the post-restitution coefficient is evaluated as

$$
c_{r}^{\text {post }}=-\frac{\dot{\delta}_{\text {measured }}^{(+)}}{\dot{\delta}^{(-)}}
$$




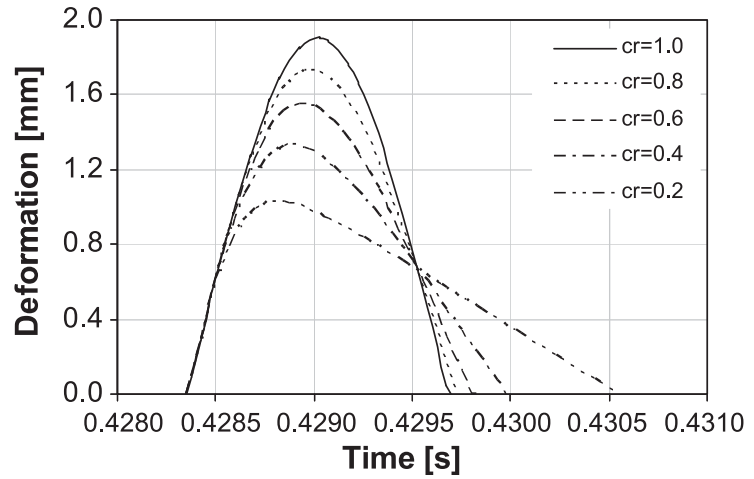

(a)

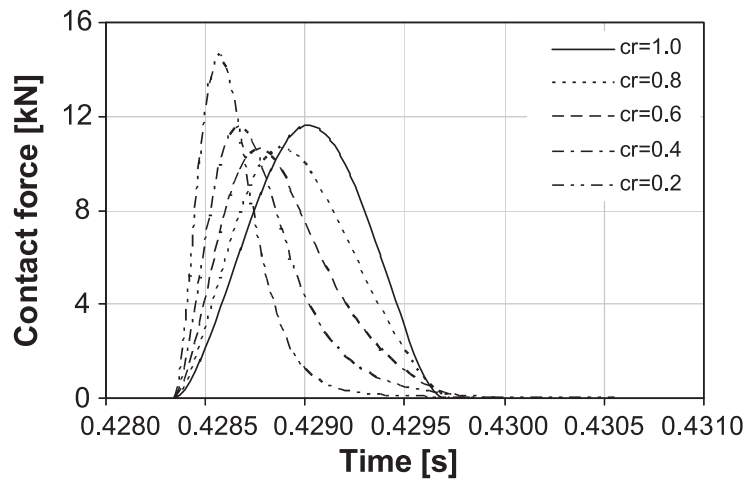

(c)

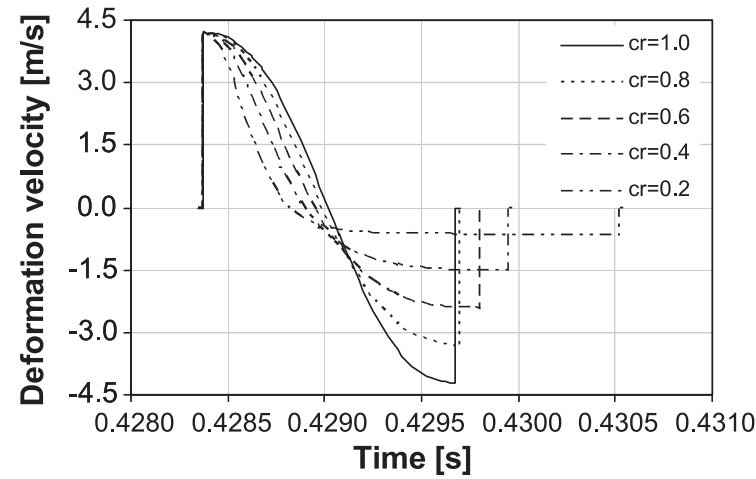

(b)

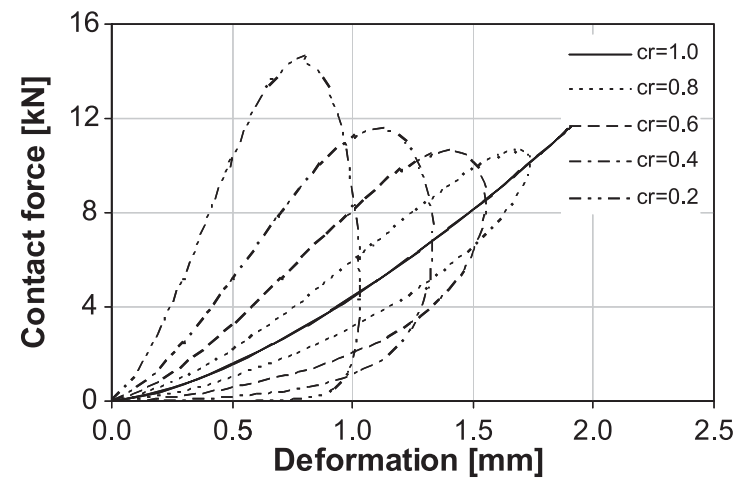

(d)

Fig. 6 Influence of the coefficient of restitution on the bouncing ball behavior: (a) Deformation versus time; (b) Velocity of deformation versus time; (c) Contact force versus time; (d) Contact force-deformation relation

Fig. 7 Relation between the post and pre-restitution coefficients

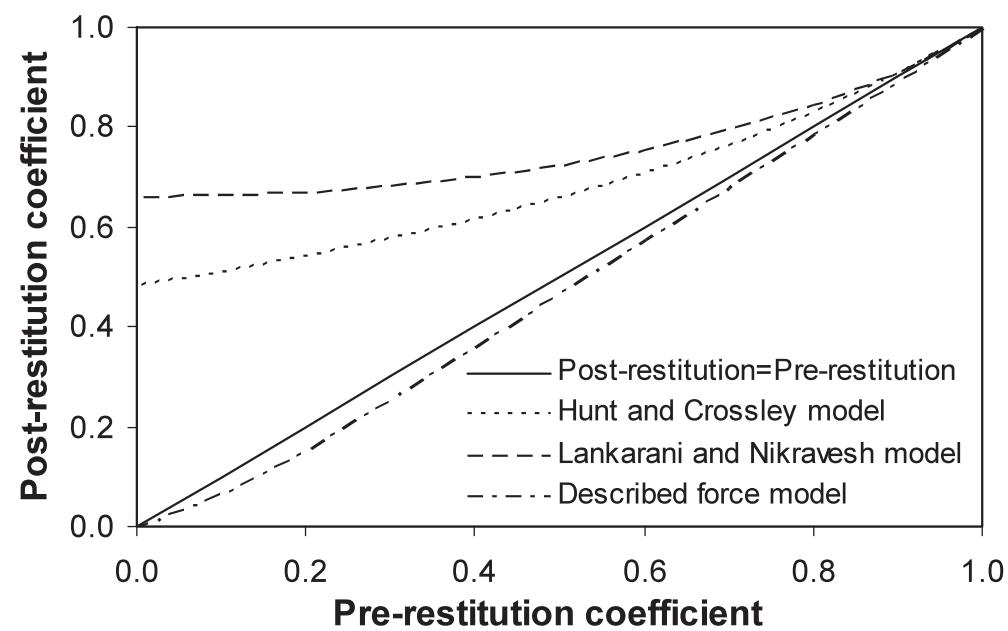

which differs from the coefficient of restitution provided as input to the contact force models hereafter referred as pre-restitution. Figure 7 illustrates the plots of the post and prerestitution coefficients for the three contact force models considered in the present work. The straight line that corresponds to the ratio between post- and prerestitution coefficients equal to the unity can be used as reference. From the plots of Fig. 7, it can be drawn that the Hunt and Crossley and the Lankarani and Nikravesh force models are valid for high values of the coefficient of restitution, as it was stated in reference [33]. Furthermore, it can also be observed that when the coefficient of restitution is null, these two force models do not reflect a perfectly plastic collision. Conversely, the contact force model described in the present work exhibits close approximation for the entire interval between 0 and 1. 
In order to better understand what are the consequences in terms of the dynamic system response when different contact force models are used, let consider a particular bouncing problem in which the ball falls down due to gravity action and impacts with a table. The table has a mass equal to $1.0 \mathrm{~kg}$ and is animated by a harmonic excitation given by

$$
y_{t}(t)=0.5+0.1 \sin 7.5 t
$$

The occurrence of contact between ball and table is determined by evaluating the relative penetration at any time during the numerical solution of the dynamic equation of motion as

$$
\delta=y_{b}-y_{t}-R
$$

where $y_{b}$ and $y_{t}$ represent the $y$ coordinate of the ball and table centers of mass, respectively, and $R$ is the radius of the ball.

In a simple way, the ball and table approach each other with a nonzero relative velocity in the normal direction until the distance between them has been vanished. Then two different scenarios can occur, namely: (i) the ball and table separate immediately after the collision with a finite positive normal velocity, (ii) or the ball and table may remain in permanent contact. The system response scenario depends on the constitutive contact law used to model the contact event, on the value of restitution coefficient and on the overall system dynamics.

Figure 8 shows the ball motion when the contact is modeled with the pure Hertz law given by (1). By analyzing the system response, it can be observed that the ball rebounds after each impact with the table. When the impact occurs in the ascendant phase of the table motion, the ball gains energy and reaches a higher position than the one before the impact.

The influence of the use of three different dissipative contact force models on the ball motion is illustrated in the plots of Fig. 9. Three difference values for the coefficient of restitution are considered, namely 0.9, 0.6, and 0.3. From the plots of Fig. 9, it can be concluded that the ball losses energy in each impact with the table. Furthermore, after some finite number of impacts the ball remains in contact with the table. This observation is valid for all dissipative contact force models used. For high values of the restitution coefficient, the Hunt and Crossley model exhibits a response similar to the case simulated with the Lankarani and Nikravesh model. The system response presents less and lower intensity rebounds when the described force approach is considered, being the permanent contact between the ball and table reached faster. This effect is more evident for lower values of the coefficient of restitution, as it can be observed in Fig. 9c. In general, it can be drawn that the Hunt and

Fig. 8 Bouncing ball response for the Hertz contact force model

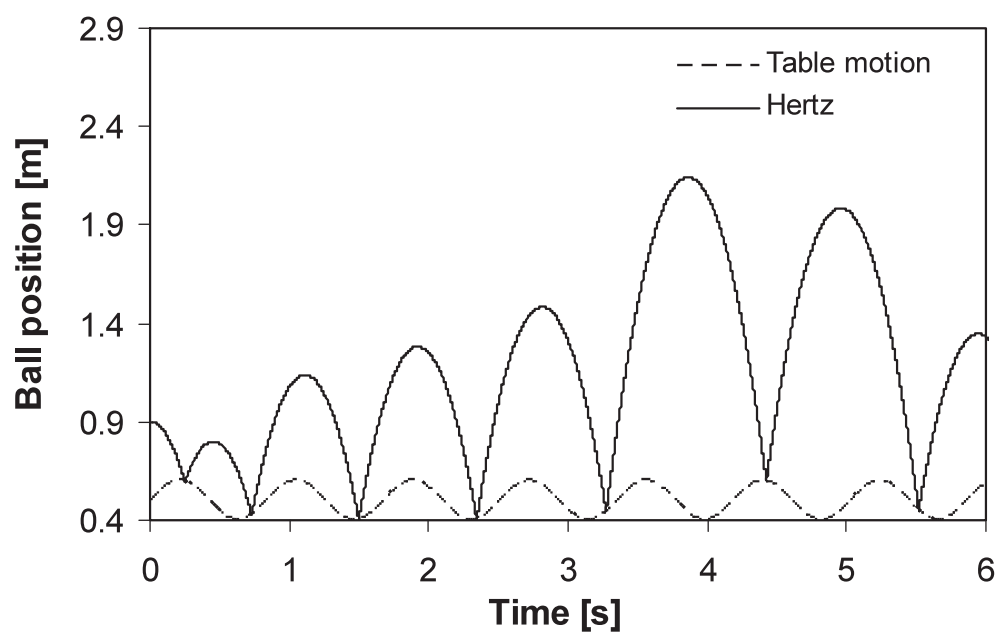


Fig. 9 Bouncing ball response for the three dissipative contact force models: (a) $c_{r}=0.9$; (b) $c_{r}=0.6$; (c) $c_{r}=0.3$

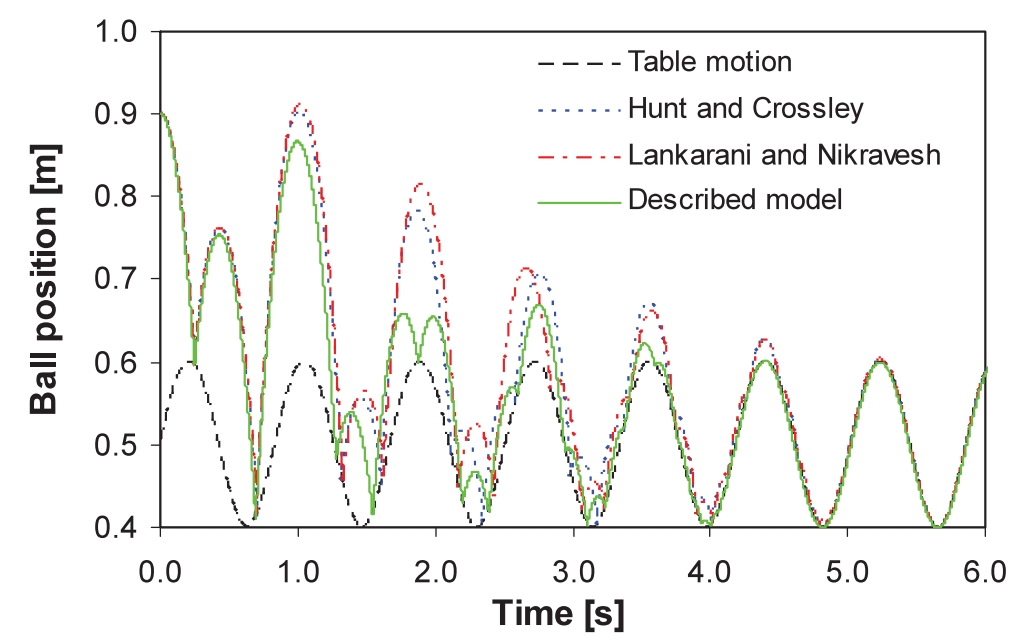

(a)

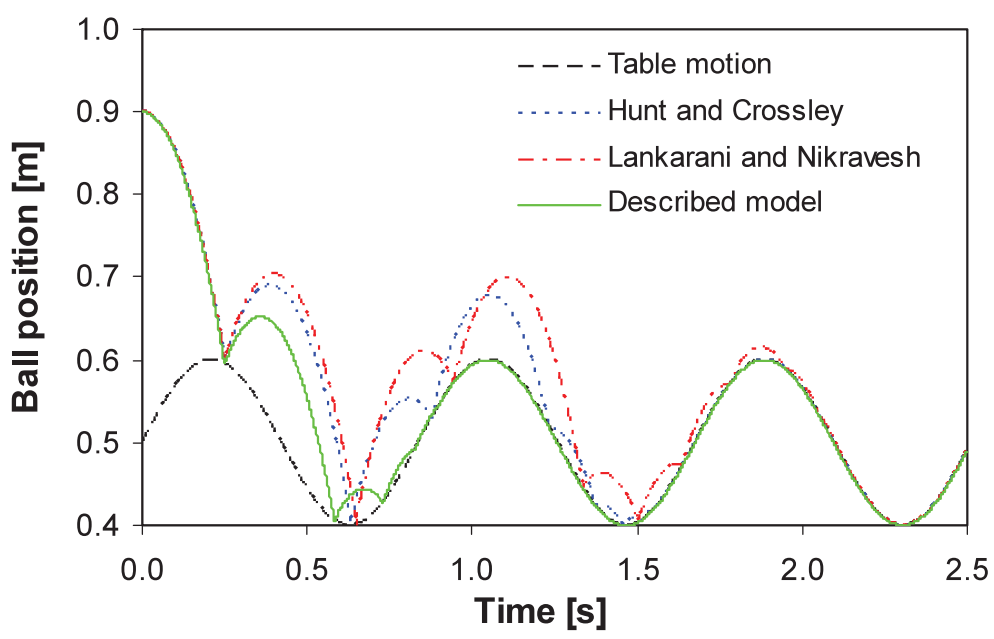

(b)

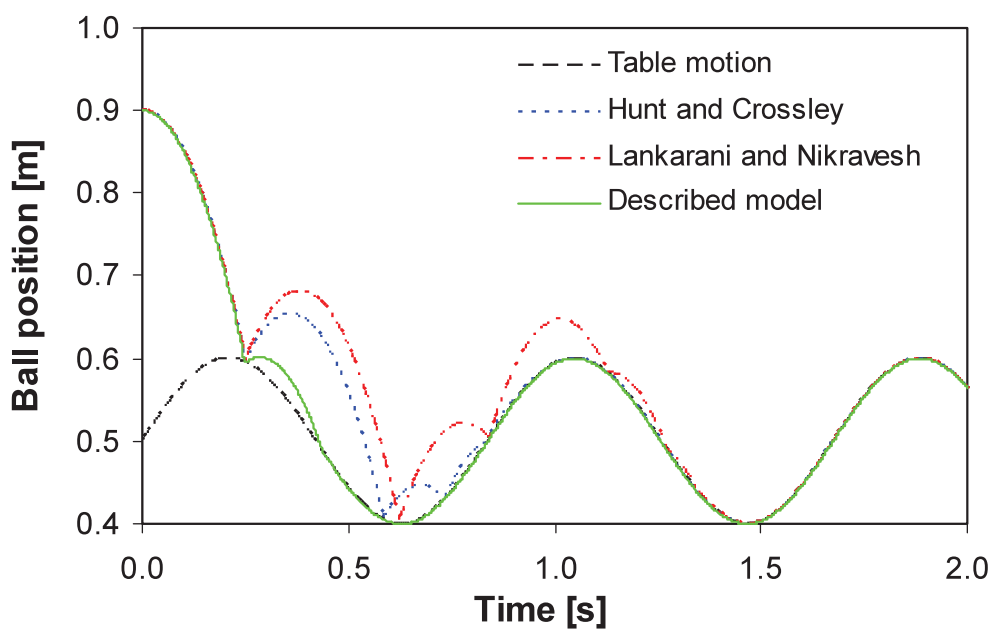

(c)

Crossley and Lankarani and Nikravesh contact force models overestimate the rebounding energy, when compared with the contact force model described in the present work.

In conclusion, for a perfectly elastic impact $\left(c_{r}=1\right)$, the three dissipative contact force models used reduce to the general Hertzian elastic model given by (1), because the hysteresis damping factor assumes a zero value. However, for a perfectly plastic impact $\left(c_{r}=0\right)$, the 
hysteresis damping factor given by Hunt and Crossley and Lankarani and Nikravesh force models does not assume an infinite value. In sharp contrast, when the described model is considered, the hysteresis damping factor tends to infinity and, consequently, the normal contact force tends also to infinity. This case makes sense from physical point of view, in the measure that for perfectly plastic impacts, the bodies will be in permanent contact after the collision, which means that the force necessary to separate them is infinite, as predicted by the force model described throughout this work. Finally, an experimental work, such as the one presented by Shivaswamy [34], must be developed in order to validate the model described here.

\section{Conclusions}

The problem of continuous contact force models for soft materials in multibody dynamics has been analyzed throughout this work. In the sequel of the process, the main issues associated with the fundamental contact mechanics were revised. Furthermore, some of the limitations and drawbacks of the classical contact force models commonly used when modeling contact events in multibody systems and robotics were discussed, also. In particular, the case in which the restitution coefficient exhibits low or medium value was analyzed. With the intent to overcome those drawbacks, a new approach for a continuous contact force was described, resulting in a mathematical expression that can be applied in the entire range of the coefficient of restitution, that is, in the cases of soft and hard contacts. This approach is straightforward to be implement in any multibody code for dynamic analysis, expresses a dependence on the material, mechanical, geometrical, and kinematical properties of the contacting bodies. In addition, it contributes to the stable numerical resolution of the equations of motion of constrained multibody systems. Finally, a simple problem was used to analyze and compared the different continuous contact force models includes in the present study, where the limits of application of each model was shown.

Acknowledgements The authors would like to thank the Portuguese Foundation for Science and Technology (FCT) for the support given through projects PROPAFE-Design and Development of a Patello-Femoral Prosthesis (PTDC/EME-PME/67687/2006), DACHOR-Multibody Dynamics and Control of Hybrid Active Orthoses (MIT-Pt/BSHHMS/0042/2008) and BIOJOINTS_-Development of advanced biological joint models for human locomotion biomechanics (PTDC/EME-PME/099764/2008). The authors want also to express their sincere gratitude to Professor Rui Pereira, from Department of Mathematics of the University of Minho, for his help in the resolution of some mathematical and numerical problems included in the present work. The second author expresses her gratitude to FCT for the Ph.D. grant SFRH/BD/40164/2007.

\section{Appendix: Integral solution of the dissipated energy due to internal damping}

This appendix contains the detailed resolution of the integral that defines the amount of energy dissipated during a contact event in terms of internal damping. For this purpose, let us rewrite (36)

$$
\Delta E=\Delta E_{c}+\Delta E_{r}=\chi\left(\dot{\delta}^{(-)}+\left|\dot{\delta}^{(+)}\right|\right) \int_{0}^{\delta_{\max }} \delta^{\frac{3}{2}} \overline{1-\left(\frac{\delta}{\delta_{\max }}\right.} \mathrm{d} \delta
$$

Now, a substitution of variable must be done according to the following relation:

$$
x=\frac{\delta}{\delta_{\max }}
$$


Differentiating (A.2) yields

$$
\mathrm{d} x=\frac{1}{\delta_{\max }} \mathrm{d} \delta
$$

Then the lower and upper limits of integration can be obtained as follows:

$$
\begin{aligned}
x(0) & =\frac{0}{\delta_{\text {max }}}=0 \\
x\left(\delta_{\max }\right) & =\frac{\delta_{\text {max }}}{\delta_{\text {max }}}=1
\end{aligned}
$$

Taking advantage of (A.2)-(A.5), (A.1) can be rewritten in the following form:

$$
\Delta E=\chi\left(\dot{\delta}^{(-)}+\left|\dot{\delta}^{(+)}\right|\right) \delta_{\max }^{\frac{5}{2}} x_{0}^{1} x^{\frac{3}{2}} \overline{1-x^{2}} \mathrm{~d} x
$$

in which the integral term is of differential binomial type [63]

$$
x^{a}\left(\alpha+\beta x^{b}\right)^{c} \mathrm{~d} x
$$

where $a, b, c, \alpha$, and $\beta$ are given constants. In the case of (A.6), these constants assume the following values $a=3 / 2, b=2, c=1 / 2, \alpha=1$, and $\beta=-1$.

According to Piskounov [63], the integral given by (A.7) together with the above values for $a, b, c, \alpha$, and $\beta$ cannot be solved analytically because it is not possible to express the integral in terms of elementary functions. Consequently, a numerical procedure must be adopted. In the present work, the trapezoidal rule is chosen to obtain the solution of this integral, which can be written as [64]

$$
{ }_{x_{0}}^{x_{n}} y(x) \mathrm{d} x \simeq \frac{x_{n}-x_{0}}{2 n}\left(y_{0}+2 y_{1}+2 y_{2}+\cdots+y_{n}\right)
$$

This integral can easily be solved by using the following Matlab implementation:

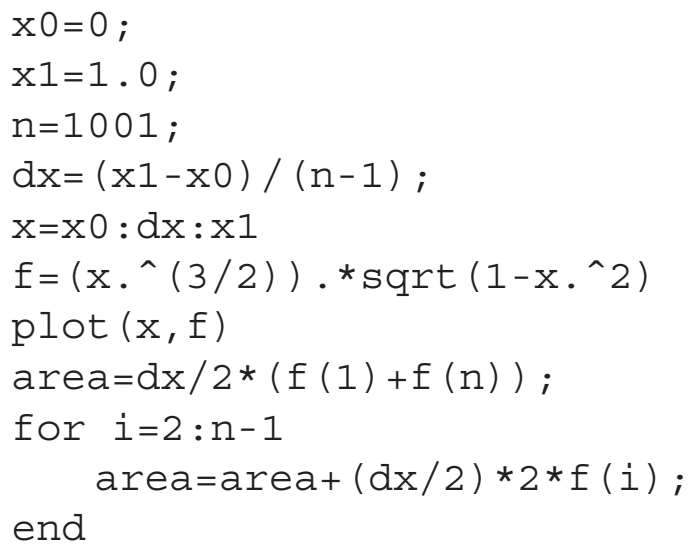

area

Finally, the result of the integral that appears in (A.6) is obtained by performing the computational simulation of the above procedure, yielding area $=0.24971047130972 \simeq$ $1 / 4$. 


\section{References}

1. Stolarsky, T.A.: Tribology in Machine Design. Butterworth-Heinemann, Stoneham-London (1990)

2. Flores, P., Ambrósio, J., Claro, J.C.P., Lankarani, H.M.: Kinematics and Dynamics of Multibody Systems with Imperfect Joints: Models and Case Studies. Lecture Notes in Applied and Computational Mechanics, vol. 34. Springer, Berlin (2008)

3. Johnson, K.L.: One hundred years of Hertz contact. Proc. Inst. Mech. Eng. 196, 363-378 (1982)

4. Goldsmith, W.: Impact, The Theory and Physical Behaviour of Colliding Solids. Edward Arnold, Sevenoaks (1960)

5. Brach, R.M.: Mechanical Impact Dynamics, Rigid Body Collisions. Wiley, New York (1991)

6. Pfeiffer, F., Glocker, C.: Multibody Dynamics with Unilateral Contacts. Wiley, New York (1996)

7. Johnson, K.L.: Contact Mechanics. Cambridge University Press, Cambridge (1999)

8. Stronge, W.J.: Impact Mechanics. Cambridge University Press, Cambridge (2000)

9. Wriggers, P.: Computational Contact Mechanics, 2nd edn. Springer, Berlin (2006)

10. Hippmann, G.: An algorithm for compliant contact between complexly shaped bodies. Multibody Syst. Dyn. 12, 345-362 (2004)

11. Gonthier, Y., McPhee, J., Lange, C., Piedboeuf, J.-C.: A regularized contact model with asymmetric damping and dwell-time dependent friction. Multibody Syst. Dyn. 11, 209-233 (2004)

12. Flores, P., Ambrósio, J., Claro, J.P.: Dynamic analysis for planar multibody mechanical systems with lubricated joints. Multibody Syst. Dyn. 12, 47-74 (2004)

13. Sharf, I., Zhang, Y.: A contact force solution for non-colliding contact dynamics simulation. Multibody Syst. Dyn. 16, 263-290 (2006)

14. Sousa, L., Veríssimo, P., Ambrósio, J.: Development of generic multibody road vehicle models for crashworthiness. Multibody Syst. Dyn. 19, 133-158 (2008)

15. Djerassi, S.: Collision with friction; Part A: Newton's hypothesis. Multibody Syst. Dyn. 21, 37-54 (2009)

16. Djerassi, S.: Collision with friction; Part B: Poisson's and Stronge's hypotheses. Multibody Syst. Dyn. 21, 55-70 (2009)

17. Bowling, A., Flickinger, D.M., Harmeyer, S.: Energetically consistent simulation of simultaneous impacts and contacts in multibody systems with friction. Multibody Syst. Dyn. 22, 27-45 (2009)

18. Dopico, D., Luaces, A., Gonzalez, M., Cuadrado, J.: Dealing with multiple contacts in a human-in-theloop application. Multibody Syst. Dyn. doi:10.1007/s11044-010-9230-y (2011)

19. Ambrósio, J., Veríssimo, P.: Improved bushing models for general multibody systems and vehicle dynamics. Multibody Syst. Dyn. 22, 341-365 (2009)

20. Mukras, S., Kim, N.H., Mauntler, N.A., Schmitz, T.L., Sawyer, W.G.: Analysis of planar multibody systems with revolute joint wear. Wear 268(5-6), 643-652 (2010)

21. Choi, J., Ryu, H.S., Kim, C.H., Choi, J.H.: An efficient and robust contact algorithm for a compliant contact force model between bodies of complex geometry. Multibody Syst. Dyn. 23, 99-120 (2010)

22. Flores, P., Leine, R., Glocker, C.: Modeling and analysis of planar rigid multibody systems with translational clearance joints based on the non-smooth dynamics approach. Multibody Syst. Dyn. 23, 165-190 (2010)

23. Gilardi, G., Sharf, I.: Literature survey of contact dynamics modeling. Mech. Mach. Theory 37, 12131239 (2002)

24. Shabana, A.A.: Dynamics of Multibody Systems. Wiley, New York (1989)

25. Ryan, R.R.: ADAMS-Multibody System Analysis Software, Multibody Systems Handbook. Springer, Berlin (1990)

26. Smith, R.C., Haug, E.J.: DADS-Dynamic Analysis and Design System, Multibody Systems Handbook. Springer, Berlin (1990)

27. Visual NASTRAN 4D, MSC Software (2002)

28. Lee, T.W., Wang, A.C.: On the dynamics of intermittent-motion mechanisms, part 1: dynamic model and response. J. Mech. Transm. Autom. Des. 105, 534-540 (1983)

29. Jackson, R.L., Green, I., Marghitu, D.B.: Predicting the coefficient of restitution of impacting elasticperfectly plastic spheres. Nonlinear Dyn. 60(3), 217-229 (2010)

30. Barkan, P.: Impact design. In: Mechanical Design and Systems Handbook, McGraw-Hill, New York (1974). Section 31

31. Hunt, K.H., Crossley, F.R.E.: Coefficient of restitution interpreted as damping in vibroimpact. J. Appl. Mech. 7, 440-445 (1975)

32. Lankarani, H.M., Nikravesh, P.E.: Continuous contact force models for impact analysis in multibody systems. Nonlinear Dyn. 5, 193-207 (1994)

33. Lankarani, H.M., Nikravesh, P.E.: A contact force model with hysteresis damping for impact analysis of multibody systems. J. Mech. Des. 112, 369-376 (1990) 
34. Shivaswamy, S.: Modeling contact forces and energy dissipation during impact in multibody mechanical systems. Ph.D. Dissertation, Wichita State University, Wichita, Kansas (1997)

35. Machado, M., Flores, P., Claro, J.C.P., Ambrósio, J., Silva, M., Completo, A., Lankarani, H.M.: Development of a planar multibody model of the human knee joint. Nonlinear Dyn. 60(3), 459-478 (2010)

36. Meireles, S., Completo, A., Simões, J.A., Flores, P.: Strain shielding in distal femur after patellofemoral arthroplasty under different activity conditions. J. Biomech. 43(3), 477-484 (2010)

37. Bei, Y., Fregly, B.J.: Multibody dynamic simulation of knee contact mechanics. Med. Eng. Phys. 26, 777-789 (2004)

38. Lin, Y.-C., Walter, J.P., Banks, S.A., Pandy, M.G., Fregly, B.J.: Simultaneous prediction of muscle and contact forces in the knee during gait. J. Biomech. 43, 945-952 (2010)

39. Burgin, L.V., Aspen, R.M.: Impact testing to determine the mechanical properties of articular cartilage in isolation and on bone. J. Mater. Sci., Mater. Med. 19, 703-711 (2008)

40. Piazza, S.J., Delp, S.L.: Three-dimensional dynamic simulation of total knee replacement motion during a step-up task. J. Biomech. Eng. 123, 599-606 (2001)

41. Silva, P.C., Silva, M.T., Martins, J.M.: Evaluation of the contact forces developed in the lower limb/orthosis interface for comfort design. Multibody Syst. Dyn. 24, 367-388 (2010)

42. Lankarani, H.M.: Canonical equations of motion and estimation of parameters in the analysis of impact problems. Ph.D. Dissertation, University of Arizona, Tucson, Arizona (1988)

43. Ye, K., Li, L., Zhu, H.: A note on the Hertz contact model with nonlinear damping for pounding simulation. Earthquake Eng. Struct. Dyn. 38, 1135-1142 (2009)

44. Hertz, H.: On the contact of solids - on the contact of rigid elastic solids and on hardness. In: Miscellaneous Papers, pp. 146-183. Macmillan and Co., London (1896) (Translated by D.E. Jones and G.A. Schott)

45. Timoshenko, S.P., Goodier, J.N.: Theory of Elasticity. McGraw Hill, New York (1970)

46. Khulief, Y.A., Shabana, A.A.: A continuous force model for the impact analysis of flexible multibody systems. Mech. Mach. Theory 22, 213-224 (1987)

47. Marhefka, D.W., Orin, D.E.: A compliant contact model with nonlinear damping for simulation of robotic systems. IEEE Trans. Syst. Man Cybern., Part A, Syst. Humans 29(6), 566-572 (1999)

48. Bibalan, P.T., Featherstone, R.: A study of soft contact models in simulink. In: Proceedings of the Australasian Conference on Robotics and Automation (ACRA), 2-4 December 2009, Sydney, Australia (2009). 8 p.

49. Flores, P.: Modeling and simulation of wear in revolute clearance joints in multibody systems. Mech. Mach. Theory 44(6), 1211-1222 (2009)

50. Tian, Q., Zhang, Y., Chen, L., Flores, P.: Dynamics of spatial flexible multibody systems with clearance and lubricated spherical joints. Comput. Struct. 87(13-14), 913-929 (2009)

51. Flores, P., Ambrósio, J., Claro, J.C.P., Lankarani, H.M.: Influence of the contact-impact force model on the dynamic response of multibody systems. Proc. Inst. Mech. Eng., Proc. Part K, J. Multi-Body Dyn. 220(1), 21-34 (2006)

52. Tian, Q., Zhang, Y., Chen, L., Yang, J.: Simulation of planar flexible multibody systems with clearance and lubricated revolute joints. Nonlinear Dyn. 60(4), 489-511 (2010)

53. Ambrósio, J., Veríssimo, P.: Sensitivity of a vehicle ride to the suspension bushing characteristics. J. Mech. Sci. Technol. 23, 1075-1082 (2009)

54. Beer, F.B., Johnston, E.R.: Vector mechanics for engineers. Statics and Dynamics (1997)

55. Greenwood, D.T.: Principles of Dynamics. Englewood Cliffs, Prentice Hall (1965)

56. Maw, N., Barber, J.R., Fawcett, J.N.: The oblique impact of elastic spheres. Wear, 101-114 (1975)

57. Zukas, J.A., Nicholas, T., Greszczuk, L.B., Curran, D.R.: Impact Dynamics. Wiley, New York (1982)

58. Hartog, J.P.: Mechanical Vibrations. Dover, New York (1985)

59. Steidel, R.F.: An Introduction to Mechanical Vibrations, 3rd edn. Wiley, New York (1989)

60. Flores, P.: Contact-impact analysis in multibody systems based on the nonsmooth dynamics approach. Post Doctoral Report, ETH-Zurich, Switzerland (2009)

61. Flores, P., Ambrósio, J.: On the contact detection for contact-impact analysis in multibody systems. Multibody Syst. Dyn. 24(1), 103-122 (2010)

62. Flores, P.: MUBODYNA-A FORTRAN program for dynamic analysis of planar multibody systems. University of Minho, Guimarães, Portugal (2010)

63. Piskounov, N.: Cálculo Diferencial e Integral. Edições Lopes da Silva, Porto, Portugal (1990)

64. Atkinson, K.A.: An Introduction to Numerical Analysis, 2nd edn. Wiley, New York (1989) 